\title{
On the Composition of the Top Layer of Microphase Separated Thin PS-PEO Films
}

\author{
Chiara Neto, ${ }^{*},+*$ Michael James, ${ }^{\perp, \S}$ and Andrew M. Telford ${ }^{\dagger}$ \\ ${ }^{\dagger}$ School of Chemistry, The University of Sydney, NSW 2006, Australia, ${ }^{\star}$ Department of Applied Mathematics, \\ Research School of Physical Sciences and Engineering, Australian National University, ACT 0200 Australia, \\ ${ }^{\perp}$ School of Chemistry, The University of New South Wales, Sydney, NSW, 2052, Australia, and ${ }^{\S}$ Bragg \\ Institute, Australian Nuclear Science and Technology Organisation (ANSTO), PMB 1, Menai, NSW 2234, \\ Australia
}

Received March 30, 2009; Revised Manuscript Received May 28, 2009

\begin{abstract}
The topography and surface composition of thin films (ca. $100 \mathrm{~nm}$ ) of a polystyrene- $b$-poly(ethylene oxide) (PS-PEO) block copolymer are investigated using a suite of complementary techniques, namely tapping mode atomic force microscopy (AFM), optical microscopy, X-ray photoelectron spectroscopy (XPS), neutron reflectometry, and wettability measurements. The copolymer films separate into lamellar structures oriented parallel to the silicon substrate, and bicontinuous and island/hole morphologies characteristic of this arrangement appear. Even though the crystalline topography of the film's surface and its wettability properties suggest the presence of PEO on the top surface, XPS and neutron reflectometry data point undoubtedly to the presence of a top layer of PS at the air/film interface. Tapping mode AFM images unequivocally demonstrate that in air only one block is present at the air/film interface. Neutron reflectometry data identify the nature of each phase-separated layer within the film. This finding differs from a model of domain arrangement proposed in a classic and much-cited paper on these systems (Macromolecules 1979, 12,323). After exposure to water, PEO blocks rearrange and access the top surface of the film. After many hours of thermal annealing, both PS and PEO blocks can be made to appear at the film/air interface, within isolated droplets formed upon film dewetting.
\end{abstract}

\section{Introduction}

Pattern formation in thin films of diblock copolymers has been the subject of much research during the past two decades. ${ }^{1-4}$ In particular, thin films of diblock copolymers have received great attention for their potential of producing templates for large area patterned surfaces for various nanofabrication applications, such as templates for inorganic composites, suboptical lithographic masks, ${ }^{5}$ photonic materials, and high-density magnetic recording devices. ${ }^{6-8}$ The study of surface properties of polymeric materials is of great research interest because of their importance in applications such as biomaterials and coatings. In particular, block copolymers containing polyethylene oxide (PEO) are often proposed as ideal biomaterials, as PEO is very effective in the prevention of protein adsorption and platelet adhesion. ${ }^{9,10}$ The combination of patterning ability and biological compatibility has made block copolymers of PEO among the most interesting and promising materials for the design of biointerfaces. Therefore, a fundamental understanding of the behavior of such copolymers at interfaces is essential.

A wide range of structures is observed in thin films of block copolymers when the blocks spontaneously microphase separate in nanodomains, such as lamellar, spherical, and cylindrical structures, depending on copolymer composition, film thickness, and sample preparation. ${ }^{2,11-24}$ In copolymers that form lamellae parallel to the substrate, a multilayered structure is formed, where the film thickness is quantized in terms of the bulk lamellar period, $L$. When one block segregates to both the substrate and the air interfaces (symmetric wetting), the film is smooth if its

*Corresponding author: Telephone: +61 29351 2752. Fax: +61 2 93513329. E-mail: c.neto@chem.usyd.edu.au. thickness is $h=n \cdot L$, where $n$ is an integer. Conversely, if one block segregates to the substrate and the other to the air interface (asymmetric wetting), smooth films are attained if the film thickness is $h=(n+1 / 2) \cdot L$. This arrangement is depicted in the cartoon in Figure 8a. If the thickness of the prepared film is not commensurate with $L$, holes or islands with a step height of $L$ form on the top surface, as indicated in Figure $8 b^{25,26}$

Here, we are particularly interested in resolving the question of the composition and topography of the top surface of thin polystyrene-poly(ethylene oxide) (PS-PEO) films supported on silicon substrates. This question is important from a fundamental scientific point of view, but it is also significant for applications, because copolymers containing PEO are often being used as biocompatible coatings, and the ability of its films to repel proteins depends crucially on the composition of the top surface. ${ }^{27,28}$

Few publications have investigated specifically this aspect. ${ }^{12,29-31}$ It is well-known that a PEO layer is in contact with the supporting silicon substrate, given its higher polarity respect to PS. However, the situation is not as clear for the film/air interface. The overwhelming majority of studies show that the component with the lowest surface energy preferentially segregates to the free surface. ${ }^{14,17,21,32,33}$ However, in the case of PS-PEO the values of surface tension are close for the two blocks $\left(\gamma_{\mathrm{PS}}=36 \mathrm{mN} / \mathrm{m}\right.$, $\gamma_{\mathrm{PEO}}=44 \mathrm{mN} / \mathrm{m}$ ), so other factors may affect the arrangement of the lamellae. For example, factors such as the crystallization of one block, the nature of the solvent, the evaporation rate, and the composition of the copolymer could drive the self-assembly process, and control the final morphology of the thin film.

The seminal paper in this field was published by Thomas and O'Malley in 1979. ${ }^{12}$ This is a classic citation in the literature, because it was one of the first studies in which angular-dependent 
X-ray photoelectron spectroscopy (XPS) was used to study the composition of thin copolymer films. The authors studied PS-PEO films cast from chloroform, a nonselective solvent for the two blocks, without any thermal annealing. Based on a model used to interpret the data, the authors concluded that, although the block with the lowest surface energy (PS) enriched the top surface of the film, both the PS and PEO block were present at the surface. The model contained blocks microphase separated into vertical domains perpendicular to the substrate, in a region of the surface at least $5 \mathrm{~nm}$ thick, with the PS block protruding higher from the top surface than the PEO block. Attempts to rationalize these findings are based on the belief that the PEO crystallization kinetics controls the surface composition, by prohibiting the amorphous PS block from migrating to the surface in sufficient quantities to completely cover the surface. ${ }^{21}$ There are indeed several instances in which crystallization of one block has been shown to drive the self-assembly process. ${ }^{34-37}$ However, the Thomas and O'Malley experiments have not been repeated, and these films were never imaged in real space. Therefore, the exact topography and composition of the top surface of the PEO-PS films spun from chloroform remains to be established.

Here, we address this problem and investigate the topography and the composition of the surface of annealed PS-PEO thin films by employing a suite of complementary techniques, namely atomic force microscopy (AFM), optical microscopy, X-ray photoelectron spectroscopy (XPS), neutron reflectometry, and wettability tests. Our results point to the need to use complementary surface techniques when studying systems as complex as these.

\section{Experimental Section}

A polystyrene- $b$-polyethylene oxide block copolymer (PS-PEO) with weight-average molecular weight of $M_{\mathrm{w}}{ }^{\mathrm{PS}}=12 \mathrm{~kg} / \mathrm{mol}$ and $M_{\mathrm{w}}{ }^{\mathrm{PEO}}=24 \mathrm{~kg} / \mathrm{mol}$ was studied. The composition is ca. $35 \%$ vol. PS and the polydispersity is approximately $M_{\mathrm{w}} / M_{\mathrm{n}}=1.05$ (Polysciences Inc. Warrington, PA). PS-PEO films were prepared by spin coating dilute $(5-16 \mathrm{mg} / \mathrm{mL})$ chloroform solutions (99+ \%, Sigma Aldrich) of the copolymer on polished silicon substrates (MEMC Electronic Materials Inc., St. Peters). The silicon wafers $(\mathrm{Si})$ are covered with a native layer of amorphous silicon oxide $\left(\mathrm{SiO}_{2}\right)$ and are very smooth. The rms roughness of the silicon substrates, determined by AFM imaging, is lower than $0.3 \mathrm{~nm}$ over an area of $5000 \mathrm{~nm} \times 5000 \mathrm{~nm}$.

Prior to spin coating, the Si wafers were thoroughly cleaned in order to reduce organic and particulate contaminants. Debris particles were eliminated using a $\mathrm{CO}_{2}$-snow jet. ${ }^{38}$ Subsequently, the wafers were exposed to sonication for a few minutes in redistilled ethanol and acetone $(99.5+\%$, Sigma-Aldrich), and treated using a radio frequency, low-temperature plasma for $30 \mathrm{~s}$ at $40 \mathrm{~W}$ (plasma reactor designed and built in-house). Cleaning and spin coating were performed inside a class-100 laminar flow cabinet. A lamellar orientation is expected for this PEO-PS copolymer, which has a sufficiently high $\chi N$ and a volume fraction of PS $f_{\mathrm{PS}}=0.35$. The phase diagram for monodisperse block copolymers presents at this composition a border between the lamellar domain, the gyroid domain, and the cylindrical domain morphology. 2,39 The prepared samples are similar in molecular weight, volume fraction, and casting solvent to sample B used in the study by Thomas and O'Malley, but no mention of overall film thickness was made there. ${ }^{12}$

The thickness of films prepared by spin coating can be controlled by varying the concentration of the copolymer solution and the spin rate. However, we note that a thickness variation of $5-10 \%$ is to be expected in each sample prepared by spin-coating, as observed previously. ${ }^{40}$ The thickness of the studied layers was determined prior to annealing by ellipsometry (Beaglehole Instruments, New Zealand), and by atomic force microscopy (AFM, Dimension 3100, Digital Instruments, Santa Barbara, CA). The presented measurements of film thickness are affected by an error of ca. 3\%. PS-PEO films of thickness between about 50 and $250 \mathrm{~nm}$ were prepared. The as-prepared spin-cast films are flat and featureless, with a rms roughness $=1.5 \mathrm{~nm}$ measured by AFM over $10 \times 10 \mu \mathrm{m}^{2}$. The absence of surface features is to be expected since the blocks are spin-cast in a disordered state, and are not in an equilibrium state. ${ }^{2,25}$ Thin films were annealed at ca. $80^{\circ} \mathrm{C}$ for different periods of time in a lab oven, and then rapidly cooled to room temperature.

A Zeiss Axioplan optical microscope equipped with a camera Axiocam MR was used for the optical micrographs. AFM was used in tapping mode to obtain high resolution images of the annealed samples. Tapping mode AFM imaging is the ideal technique to study phase separation in block copolymers, because its topographical images reveal the height profile and the morphology of the surface, and its phase images highlight variations in local properties of the surface, such as stiffness, viscoelasticity, and chemical composition, and therefore reveal the presence of different blocks at the top surface. ${ }^{41-46}$ The phase image provides a map of the phase lag of the cantilever vibration relative to the piezo drive during scanning, which depends on the energy dissipation during tip-sample interaction. Low tapping forces and relatively low scan rates were employed in this study in order to probe the true surface of the film and avoid surface indentation. ${ }^{47}$

$\mathrm{X}$-ray photoelectron spectroscopy data were obtained with an Escalab 220i-XL spectrometer (VG, UK) using monochromated Al $\mathrm{K} \alpha$ exciting radiation. Typical operating conditions were: energy $1486.6 \mathrm{eV}$, power $120 \mathrm{~W}(10 \mathrm{kV}, 12 \mathrm{~mA})$, spot size $1 \mathrm{~mm}^{2}$. By analyzing the photoemitted electrons normal to the sample's surface, the instrument analyses approximately the top 5-10 nm of the surface of the film. This instrument also allows the depth profiling of the sample, as the film is slowly removed by sputtering with a $5 \mathrm{kV}$ Argon ion beam. The average sputtering rate was calibrated on a metal surface to be ca. $0.2 \mathrm{~nm}$ per second.

Neutron reflectivity data were measured using the Platypus time-of-flight neutron reflectometer, ${ }^{48}$ and a cold neutron spectrum $(3.0 \AA \leq \lambda \leq 18.0 \AA)$ at the OPAL $20 \mathrm{MW}$ research reactor (Australian Nuclear Science and Technology Organisation, Sydney). Twenty-three $\mathrm{Hz}$ neutron pulses were generated using a disk chopper system (EADS Astrium $\mathrm{GmbH}$ ) in the medium resolution mode $(\Delta \lambda / \lambda=4 \%)$, and recorded on a 2-dimensional helium-3 neutron detector (Denex $\mathrm{GmbH}$ ). Reflected beam spectra were collected at $0.5^{\circ}$ for $2 \mathrm{~h}(0.3 \mathrm{~mm}$ slits) and $2.0^{\circ}$ for $6 \mathrm{~h}$ (1.2 $\mathrm{mm}$ slits) respectively. Direct beam measurements were collected under the same collimation conditions for $1 \mathrm{~h}$ each.

Structural parameters associated with layers within the PSPEO film were refined using the MOTOFIT package ${ }^{49}$ using neutron reflectivity data as a function of momentum transfer normal to the surface $\mathrm{Q}_{z}=(4 \pi(\sin \theta) / \lambda)$. An initial structural model was prepared using estimated values of neutron scattering length density (SLD) (Table S1), based on a mass density for the PS component of the film of $1.05 \mathrm{~g} / \mathrm{cm}^{3}$ and $1.13 \mathrm{~g} / \mathrm{cm}^{3}$ for PEO. A thin native silicon oxide layer was included in each structural model that was tested. The Levenberg-Marquardt method was used to minimize $\chi^{2}$ values.

The wetting properties of the employed materials were investigated by measuring the advancing and receding contact angles of $5-10 \mu \mathrm{L}$ droplets of MILLI-Q water with a KSV CAM200 Contact Angle System (KSV Instruments Ltd., Helsinki, Finland), and by performing condensation experiments under a reflection optical microscope. The equilibrium, advancing, and receding contact angle of water on the films were measured at three different locations on three different samples, and the average and standard deviation of these values were calculated. Condensation experiments were performed by cooling the copolymer films on an ice surface, while blowing some moist air on the copolymer film place under an optical microscope. These experiments were performed on three different copolymer film samples. 

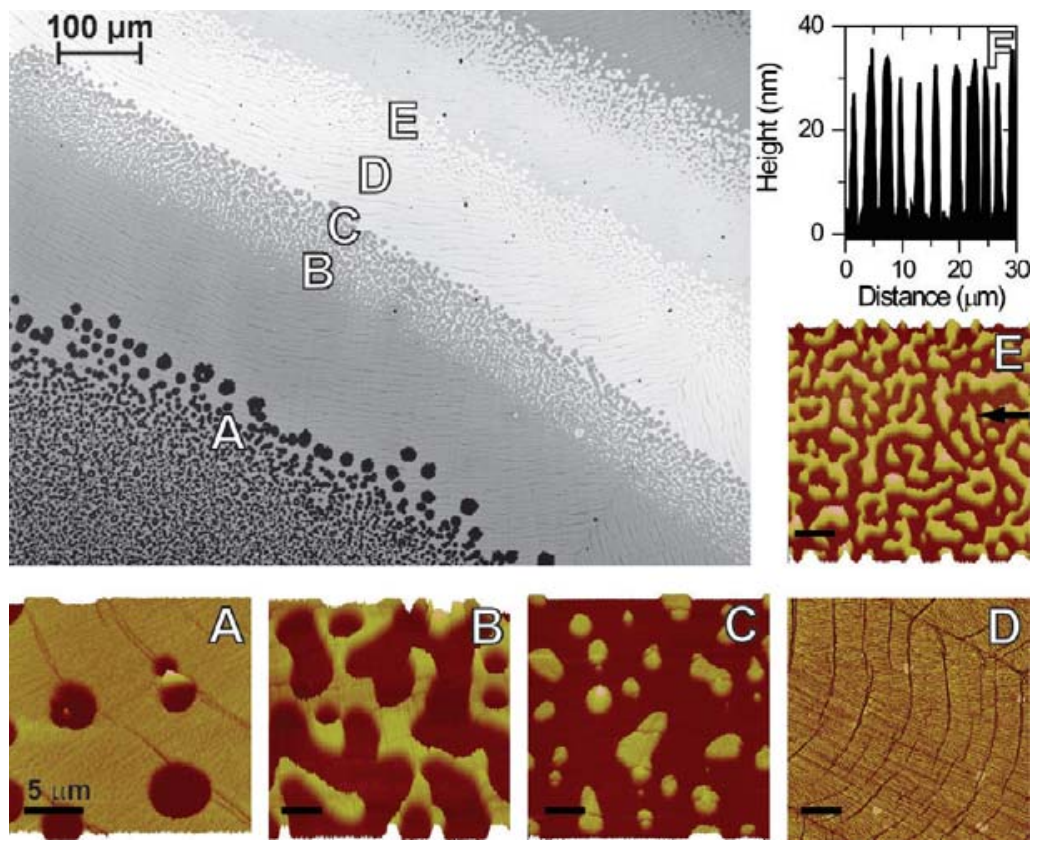

Figure 1. Summary of the morphologies observed in thin films of a PS-PEO copolymer (PS12k-PEO24k) after annealing. Main image: optical micrograph of a PS-PEO film with a gradient in thickness from the bottom left-hand corner $(130 \mathrm{~nm})$ to the top right-hand corner. The different colors indicate regions of different film thickness (from A $130 \mathrm{~nm}$, to B $102 \mathrm{~nm}$, to D $74 \mathrm{~nm}$ ). The sample was prepared by spin coating a chloroform solution onto a silicon wafer, and the gradient in thickness is produced at the edge of the substrate. The microphase separation of the copolymer is clear in this terracing effect. (A-E) Tapping mode AFM images taken on films that have similar morphologies as the regions marked with letters in the micrograph. The scale bar is $5 \mu \mathrm{m}$ in all the AFM images. (F) Cross section taken on the AFM scan in part $\mathrm{E}$ at the position of the arrow, showing that the islands are all of the same thickness, $L=28 \mathrm{~nm} \pm 2 \mathrm{~nm}$.

\section{Results}

1. Differential Scanning Calorimetry and Thermal Gravimetric Analysis. Differential scanning calorimetry (DSC) measurements performed with a Modulated DSC 2920 TA Instruments revealed that for the PS blocks in this copolymer the glass transition temperature is $T_{\mathrm{g}}=58{ }^{\circ} \mathrm{C}$ and for the crystalline PEO blocks the melting temperature is $T_{\mathrm{m}}=67^{\circ} \mathrm{C}$ (see Supporting Information, Figure S1(a)). At the annealing temperature of $80^{\circ} \mathrm{C}$ the blocks in the melt rearrange in a lamellar arrangement, determined by the balance between the enthalpic gain of demixing of the two blocks and the entropic cost of chain confinement within domains, and by the presence of the two interfaces. Since the $T_{\mathrm{g}}$ of the PS blocks is lower than the $T_{m}$ of PEO crystals, as the film cools down, the PEO blocks crystallize within a relatively fluid matrix of the amorphous PS phase.

Thermal gravimetric analysis (TGA) was performed with a TA Instruments Hi-Res TGA2950. The sample was heated from room temperature to $80^{\circ} \mathrm{C}$ at a rate of $20^{\circ} \mathrm{C} / \mathrm{min}$, and then the temperature was held at $80^{\circ} \mathrm{C}$ for $50 \mathrm{~min}$. The trace shown in Figure S1(b) reveals a very small weight loss, ca. $0.4 \%$ upon heating, and there are no signs of degradation of the copolymer in this temperature range. If the heating procedure is repeated, no additional weight loss can be measured, which indicates that the small loss of weight is due to the loss of water.

2. Optical and AFM Imaging. Thermal annealing of the PS-PEO films induced the appearance of the lamellar microphase separation, with the characteristic island/hole morphologies on the surface of the film. The main large image in Figure 1 is an optical micrograph taken on the edge of an annealed film. The different colors in the micrographs correspond to regions of different film thicknesses, from a value of $130 \mathrm{~nm}$ in the bottom left-hand corner to a value of ca. $10 \mathrm{~nm}$ in the top right-hand corner. The terracing effect is due to the fact that the film thins down close to the edge of the substrate because of the spin coating process. ${ }^{50}$ The letters in the optical micrograph in Figure 1 mark different types of observed morphologies in the film, and the tapping mode AFM images in Figure 1 are corresponding magnifications of each morphology.

The morphologies observed in Figure 1 depend on the thickness of the film, and can be explained with commensurability effects, as discussed in the introduction. When the film thickness is not an exact multiple of the lamellar thickness $L$, incomplete lamellae form on the top surface of the film. The transition between adjacent lamellae occurs through holes, islands, and bicontinuous structures. Figure 1A corresponds to a morphology of holes in the top lamella, with surface area of the holes of ca. $20 \%$. In Figure $1 \mathrm{~B}$ the film is thinner and the surface coverage of holes has increased (ca. 70\%). Figure 1C shows a morphology of small islands emerging on the top lamella (surface coverage of the islands ca. 20\%). Figure 1D corresponds to a smooth region, where the film thickness is an exact multiple of the lamellar thickness. Finally, Figure 1E illustrates a maze-like bicontinuous pattern, and the islands occupy ca. $35 \%$ of the surface. The correlation length of such bicontinuous patterns has been studied in other copolymer films before, and depends on factors such as lamellar thickness and molecular weight of the copolymer. ${ }^{51}$

The depth of the holes (Figure 1A) and the height of the islands and bicontinuous patterns (Figure 1B, C, and E) are the same and correspond to one lamellar period, $L=28 \pm$ $2 \mathrm{~nm}$. This is illustrated in the cross section in Figure $1 \mathrm{~F}$, which corresponds to a line section taken on the black arrow in Figure 1E.

Both AFM and optical images show clear evidence of crystalline orientation on the surface of the films, as shown in Figures 1 and 2. In the optical micrograph in Figure $2 a$, a 

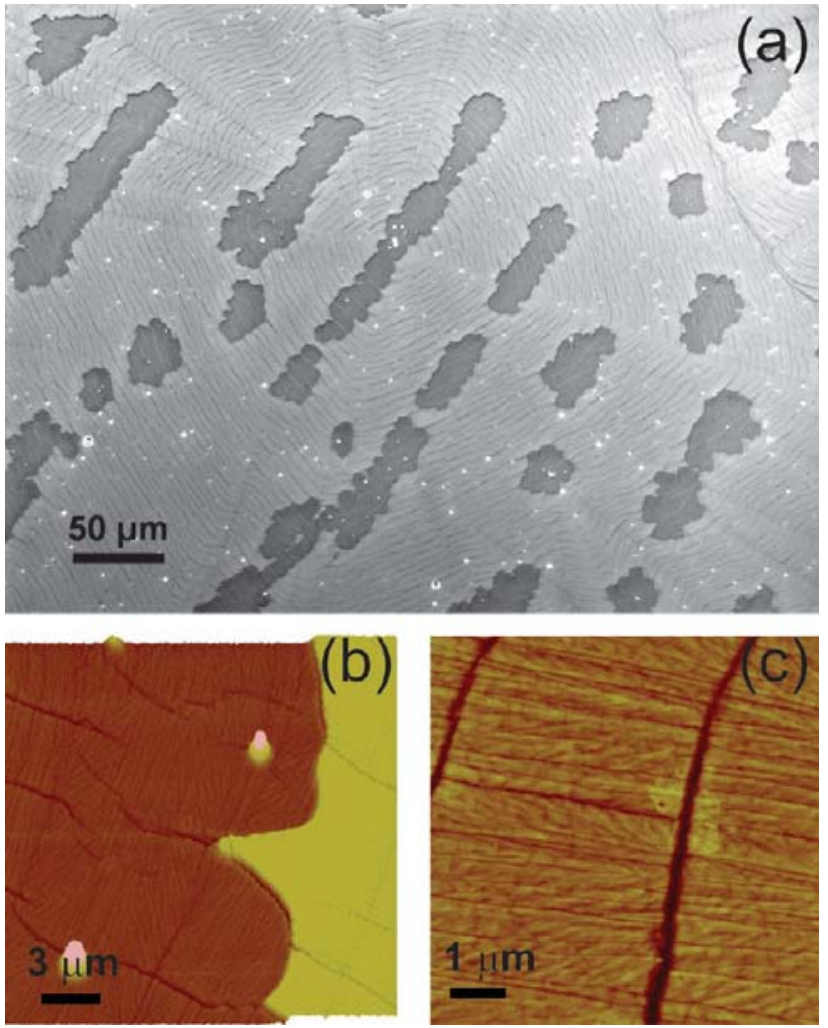

Figure 2. Crystalline orientation of the surface of a PS-PEO film (100 $\mathrm{nm}$ thickness) after $14 \mathrm{~h}$ thermal annealing. (a) Optical micrograph of the top surface of the PS-PEO film. (b) Tapping mode AFM image of the edge of a hole on the surface of the same film as in part a. The crystal orientation is the same on the bottom of the hole (left side of the image) and on the top lamella (right side of the image). The vertical scale is $70 \mathrm{~nm}$. (c) Tapping mode AFM image of the top surface of the same film as in part a (top lamella). The vertical scale is $60 \mathrm{~nm}$.

crystallization center is visible just above the center of the image, and the crystal shows long-range concentric order all around this center. Elongated gray patches are present on the top surface, all aligned with the same orientation. By observing different areas of the samples, we concluded that these patches are holes in the top surface, and the orientation of these holes with respect to the substrate is determined by the spin coating process. Indeed, the holes are oriented radially from the center of the film where the copolymer solution drop is initially deposited for spin coating. This is an intrinsic characteristic of the spin coating process that produces slight variations in the film thickness.

The morphology of the surface of the annealed PS-PEO films displays prominent crystalline ordering, in the form of densely packed ridges all oriented roughly radially out from the nucleus of the crystal (Figure 2, parts a and c), as observed previously in PEO thin films. ${ }^{52}$ The crystalline orientation is consistently the same on the two contiguous top lamellae, i.e. both on the top surface and inside the holes, as illustrated in Figure 2(b) and in Figure S2 (Supporting Information). The crystallization of one PEO layer induces the crystallization of adjacent PEO layers, separated by intervening layers of the amorphous block. This can be explained assuming that there is a crystallographic connection between the PEO crystallites in adjacent layers, in the form of screw or edge dislocations. ${ }^{53,54}$

Tapping-mode AFM allows, using phase contrast, to map the lateral distribution of each block near the film surface, due to the different mechanical properties of the two

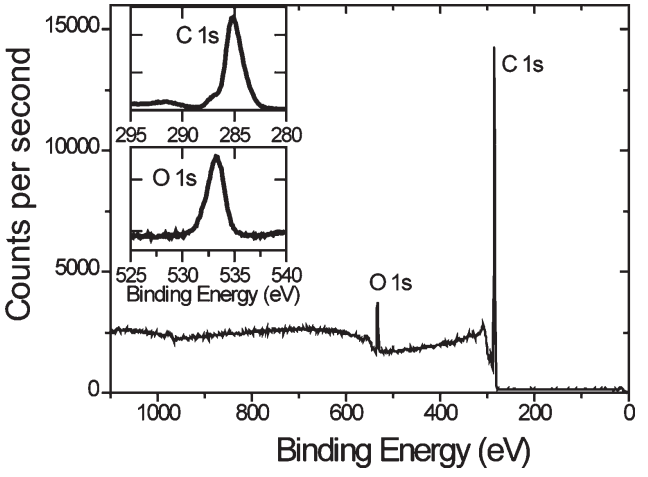

Figure 3. X-ray photoelectron spectroscopy (XPS) analysis of the surface composition of a PS-PEO thin film. The two insets are enlargements of the two main peaks in the spectrum, corresponding to $\mathrm{O} 1 \mathrm{~s}$ and $\mathrm{C} 1 \mathrm{~s}$ signals. This spectrum reveals that both the PS and the PEO blocks are near the top surface of the film, i.e. within the top $10 \mathrm{~nm}$ of the film.

blocks. ${ }^{42,43,47}$ PS-PEO copolymers have been used in the past as ideal candidates for studies of the phase contrast behavior in a AFM experiments, because of the large stiffness difference between the blocks. ${ }^{46}$ In our films, no phase contrast was registered on the microphase separated films (Figure S3). This is an unequivocal indication that only one block is present at the top surface of the film, compatibly with a microphase separation into lamellae parallel to the substrate. The AFM and optical images do not allow determining which of the two blocks is present at the top surface of the film. Since atactic PS does not crystallize, and crystalline order is clearly visible in the optical and AFM images, it would be tempting to conclude that the top crystalline layer of these films is made of the PEO block. However, the surface of the PEO crystals could also be covered by a thin overlayer of PS invisible to AFM, as for example in the case of crystalline isotactic PS. ${ }^{55}$ AFM imaging alone did not allow distinguishing between these two possible scenarios, therefore we employed other complementary techniques which are sensitive to the chemical nature of the top surface: XPS, neutron reflectometry, and wettability measurements.

3. XPS Analysis. In order to ascertain the chemical composition of the top surface of the film, we performed XPS analysis on two identical annealed PS-PEO thin films. The measured $\mathrm{C} 1 \mathrm{~s}$ and $\mathrm{O} 1 \mathrm{~s}$ core level spectra for both samples were identical, so Figure 3 and insets illustrate the results on one of the samples. These spectra are similar to data previously measured on similar films. ${ }^{12,29}$ The C $1 \mathrm{~s}$ region at a binding energy of ca. $285 \mathrm{eV}$ can be deconvoluted into two separate peaks. The main peak is attributed to the carbon in the styrene unit (and some possible hydrocarbon contaminant), while the left shoulder at $288 \mathrm{eV}$ is attributed to the $\mathrm{C}-\mathrm{O}$ ethylene unit. The smaller peak at $292 \mathrm{eV}$ clearly presents shakeup features which indicate aromatic species, i.e. polystyrene. A lower concentration of oxygen can also be seen at the surface, as shown by the peak at $533 \mathrm{eV}$. The main information that these spectra provide is that both PS and PEO are present within the top ca. $10 \mathrm{~nm}$ of the film's surface being probed.

We also performed XPS depth profiles for two samples of PS-PEO thin films of thickness $95 \mathrm{~nm}$, by sputtering away the films at a rate of $0.2 \mathrm{~nm} /$ second with a $5 \mathrm{kV}$ argon ion beam. Both data sets are shown in Figure 4, where a map of the concentration of oxygen, carbon, and silicon is shown. Figure $4 \mathrm{a}$ is an enlargement of the full spectrum at short sputtering times. In both samples, both carbon signal and an oxygen signal (ca. $5.7 \%$ of the total composition) 


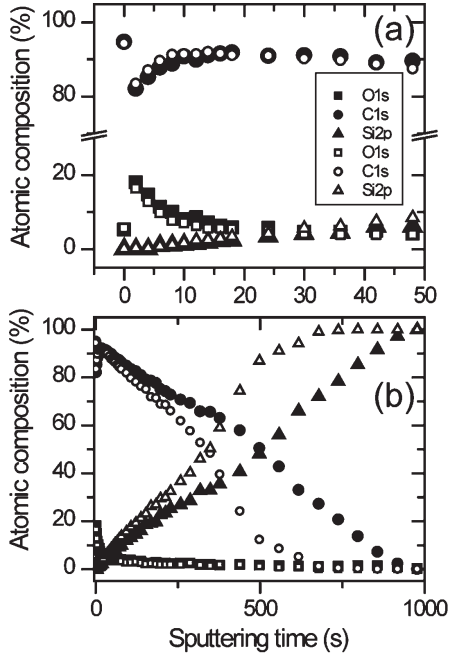

Figure 4. X-ray photoelectron spectroscopic (XPS) analysis of the depth profile composition of two PS-PEO thin films. Two sets of data relative to two different samples are presented in both graphs, with one sample corresponding to full symbols and the other sample corresponding to smaller empty symbols. Part a shows an enlargement of the region in part $\mathrm{b}$ at short sputtering times.

are observed on the top surface, as already shown in Figure 3. After the first two seconds of sputtering, the signal of the oxygen jumped up to ca. $17 \%$ of the total composition in both samples. At the same time, the shakeup features, indicative of aromatic compounds, disappeared (not shown), and the peak corresponding to the $\mathrm{C}$ $1 \mathrm{~s}$ decreased sharply to ca. $82 \%$. It is clear from the similarity of the spectra of the two different samples at short sputtering times that this feature is not an artifact of the measurement.

In the initial two seconds, a layer a few nanometers thick can be removed by the argon beam, corresponding approximately to the thickness of a molecular layer. The uncertainty in the exact amount of material removed derived from the fact that the sputtering rate was calibrated on a metal sample, rather than a PS-PEO polymer. This data shows that a thin layer of aromatic compounds coats the film, and just below this layer there is a significantly higher amount of oxygencontaining compound. The simplest interpretation is that the top layer of the film is made of PS, which lies on top of a PEO layer, which makes up the top lamella. A PS layer is present at the top surface, but its thickness cannot be determined exactly with XPS.

As illustrated in the complete depth profiles in Figure 4b, the data relative to the two samples is slightly different at longer sputtering times, but presents substantially the same information. First of all, XPS is not sufficiently sensitive to reveal the lamellar structure in the depth profile. Second, as the ion beam sputtering continues, the contribution from the oxygen within the film decreases very rapidly with time, much more rapidly than that from the carbon signal. Third, the signal from the silicon substrate gradually increases, and gradually dominates after approximately 700-1000 s of sputtering. The comparison between the two sets of data reveals that the XPS technique is not optimal in revealing information on the depth profile of polymer layers whose main distinguishing feature is the presence of oxygen. In particular, in XPS depth studies the oxygen is preferentially sputtered by the beam with respect to the other elements, and this causes a much faster removal of the oxygen. ${ }^{56}$ The one conclusion that we can draw form the XPS spectra is that

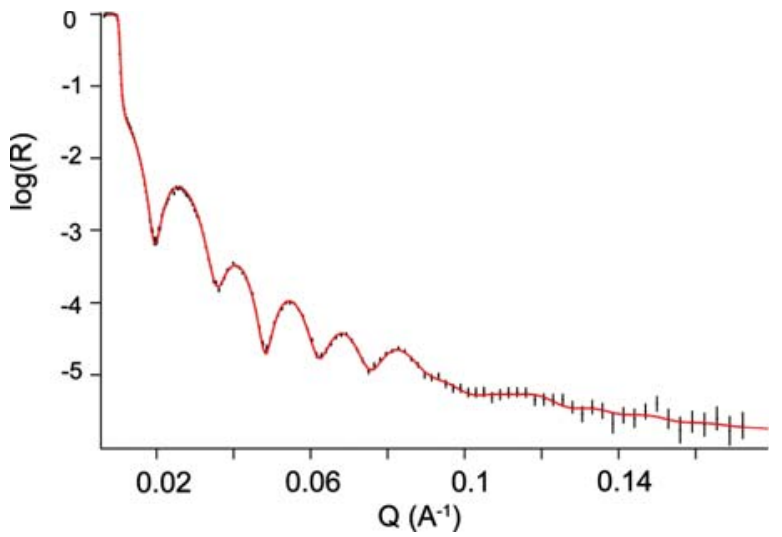

Figure 5. Neutron reflectivity data for an annealed PS-PEO film of thickness $46.8 \mathrm{~nm}$. The experimental data (points) and calculated model based upon the refined structural model (solid line) are presented together.

oxygen is hardly present in the first few nanometers of the film, but is present strongly under this top layer.

4. Neutron Reflectometry. Figure 5 shows measured and calculated neutron reflectivity data for an annealed PS-PEO film, with the solid line being the calculated reflectivity based upon a refined structural model. Preliminary Fourier transforms of the reflectivity data prior to detailed modeling suggested an overall film thickness of $47 \mathrm{~nm}$, which was used to constrain initial refinement attempts with complex multilayer models.

Various models were used to initially refine the structure of the PS-PEO film using the neutron reflectivity data. A single-layer model of constant composition (based on an estimated SLD for the copolymer) led to a very poor fit to the observed data $\left(\chi^{2}=0.054\right)$. Three- and four-layer models consisting of alternating PEO and PS layers, with a PEO layers adjacent to the $\mathrm{Si}$ substrate were also modeled and led to reasonable fits to the observed data $\left(\chi^{2}=0.0059\right.$ and $\chi^{2}=$ 0.0028 , respectively). By far the best fit $\left(\chi^{2}=0.0018\right)$ to the observed neutron data was obtained by a five-layer polymer model, illustrated Figure 8c.

Table 1 summarizes the results of the fitting of the neutron reflectivity data, namely the refined values of the layer thickness, interfacial roughness between the polymer layers, and the refined neutron SLD values. Because the overall film thickness was smaller than ${ }^{3} / 2 L$, the film presented a hole/ island morphology, which made the analysis of the neutron data more challenging.

A film similar to that studied by neutron reflectometry was imaged by AFM and showed a film thickness of $45 \pm 5 \mathrm{~nm}$, with a proportion of islands occupying about $45 \%$ of the area of the film (Figure S4). The knowledge of the morphology and general structure of the film derived from AFM enabled us to derive a suitable structural model to be used in the analysis of the neutron reflectivity data. These data (in conjunction with the structure derived from AFM and XPS) gave a very clear 5-layer model of the phase-separated polymer, and indicated clearly that a PS layer is on the top surface of the film. The film's structure is made up of a continuous PEO/PS half-lamella adjacent to the Si substrate (layers 4 and 5, thickness 15.7(1) nm), and an incomplete lamella above this, made of a bottom 6.5(1) nm PS layer, a middle 18.5(1) nm PEO layer, and an top 6.1(1) nm PS layer (layers 1-3, thickness 31.1(1) nm). The blocks in the incomplete top lamella have a reduced scattering length density, due to the presence of the holes/islands morphology in the top part of the film. The lamellar thickness derived from the 
Table 1. Refined Structural Parameters from Neutron Reflectivity Data on a PS-PEO Film of Thickness 46.8 nm

\begin{tabular}{|c|c|c|c|c|}
\hline component & structure & $\begin{array}{l}\text { layer thickness } \\
\text { (nm) }\end{array}$ & $\begin{array}{l}\text { scattering length density } \\
\qquad\left(\times 10^{6} \AA^{-2}\right)\end{array}$ & $\begin{array}{l}\text { interfacial roughness } \\
(\mathrm{nm})\end{array}$ \\
\hline 1. PS top layer & & $6.1(1)$ & $1.03(1)$ & $1.4(1)$ \\
\hline 2. PEO middle layer & incomplete lamella with hole/island morphology & $18.5(1)$ & $0.68(1)$ & $1.5(1)$ \\
\hline 3. PS bottom layer & & $6.5(1)$ & $1.24(1)$ & $1.4(1)$ \\
\hline 4. PS layer & half_lamella & $2.6(1)$ & $1.51(1)$ & $1.7(1)$ \\
\hline 5. PEO layer & half-lamella & $13.1(1)$ & $0.82(1)$ & $2.1(1)$ \\
\hline $\mathrm{SiO}_{2}$ & & $1.1(1)$ & 3.47 & $0.5(1)$ \\
\hline $\mathrm{Si}$ & & & 2.07 & $0.5(1)$ \\
\hline
\end{tabular}
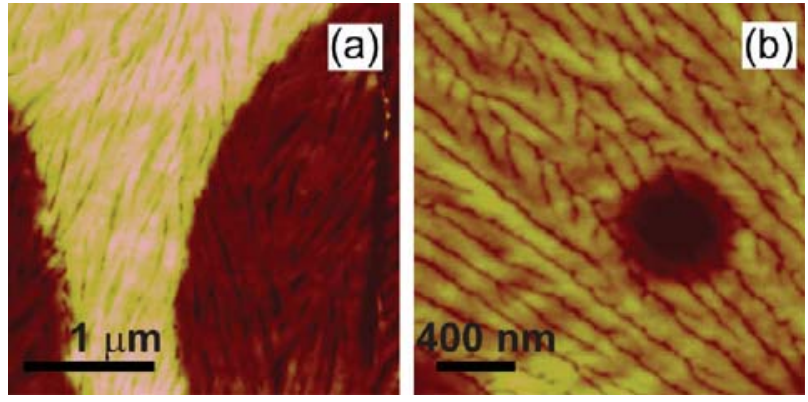

Figure 6. Tapping mode AFM images of annealed PS-PEO films exposed to MILLI-Q water for $5 \mathrm{~min}$ (a) and $24 \mathrm{~h}$ (b). The film in part $\mathrm{a}$ is $100 \mathrm{~nm}$ thick, and the film in part b is $90 \mathrm{~nm}$ thick. The vertical scale is $40 \mathrm{~nm}$ in part a and $65 \mathrm{~nm}$ in part b.

neutron data is in agreement with the values obtained by AFM. The relatively low thickness of the PS layer in the bottom half-lamella (layer 4) and the relatively large SLD value for the PEO layer (layer 5) suggest that some degree of intermixing between PS and PEO might be present in the bottom half-lamella.

5. Wettability Experiments. Contact angle measurements gave indication that the composition of the surface of the film changes upon exposure to water, as previously observed. ${ }^{57}$ The film is initially not wetted by water: the equilibrium contact angle measured upon first placing a droplet of water on the film (of thickness $95 \mathrm{~nm}$ ) is $\theta_{e q}=$ $105^{\circ} \pm 5^{\circ}$, and the value of the advancing contact angle is also the same within experimental error. These values are very close to those measured on a PS spin coated film $\left(\right.$ ca. $\left.\theta_{a d v}=99^{\circ} \pm 3^{\circ}\right) .{ }^{58}$ However, the measurement of the receding contact angle showed a surface almost completely wetted by water, with a $\theta_{\text {rec }}=15^{\circ} \pm 5^{\circ}$. This confirms previous studies that found that the surface of a PS-PEO film is hydrophobic when exposed to air, but upon exposure to water, the surface of turns rapidly hydrophilic. ${ }^{27,57}$ This behavior indicates that the PS block is present at the air/film interface, but upon exposure to water the chains of PS block move away from the water/film interface and are replaced by the more hydrophilic chains of the PEO block.

Parts $a$ and $b$ of Figure 6 show the effect of immersing PS-PEO films in MILLI-Q water for $5 \mathrm{~min}$ and $24 \mathrm{~h}$, respectively, and then drying them with a jet of nitrogen. In both cases the crystalline ordering observed on the untreated films is maintained, but the crystal fibrils are affected by the contact with water. In Figure 6a, the ridges appear more rigid, straight, and distinct from each other than before exposure to water (compare with parts b and $\mathrm{c}$ of Figure 2). In Figure 6b, the crystal orientation appears more orderly, the arrangement of the ridges more regular, and the ridges appear to have a defined average thickness of $120 \pm$ $30 \mathrm{~nm}$. These modifications are compatible with the presence of the water-soluble PEO block very near the top surface, and with the fact that its crystalline fibrils swell upon

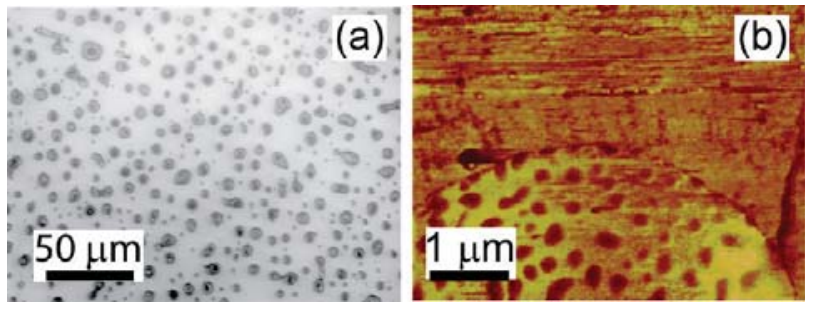

Figure 7. Droplets developed upon dewetting of a PS-PEO film of thickness $75 \mathrm{~nm}$ on a silicon substrate after a long annealing $(41 \mathrm{~h})$. (a) Optical micrograph of a dewetted PS-PEO film. The gray dots are PS-PEO droplets on the substrate. (b) Tapping mode AFM phase image of part of a PS-PEO dewetted droplet on the substrate. The phase contrast within the droplet (ca. $5 \mathrm{deg}$ ) is due to the difference in the mechanical properties of PS and PEO.

exposure to water and then recrystallize in a more orderly fashion while the film dries.

Condensation experiments were also performed on the films to reveal the possible presence of wettability contrast on the top surface. ${ }^{59}$ Water vapor droplets condensed uniformly over the surface of the film, and showed no signs of the presence of boundaries between hydrophilic and hydrophobic regions at the film surface. These measurements are qualitative in nature, but nonetheless reinforce the view that only one block is present at the film surface (Figure S5).

6. Dewetting at Long Annealing Times. After being exposed to thermal annealing for many hours, the thin PS-PEO films eventually dewet from the substrate. The duration of annealing before the onset of dewetting depends on film thickness; for films of thickness ca. $100 \mathrm{~nm}$ dewetting occurs for annealing times longer than $24 \mathrm{~h}$; for films of ca. $50 \mathrm{~nm}$, dewetting starts after ca. $10 \mathrm{~h}$. Dewetting is the process by which liquid films that do not wet their substrate break up in holes and eventually are transformed into a series of isolated droplets on the substrate. ${ }^{60,61}$ Metastable thin liquid films rupture by nucleation of holes around debris particles or impurities in the film. ${ }^{61-64}$ Dewetting in PS-PEO films is due to autophobic behavior, whereby the liquid melt does not wet a thin layer of itself, as observed in experiments on thin PEO films. ${ }^{65,66}$ The basic physical picture that explains this scenario is that of pseudopartial wetting: ${ }^{67} \mathrm{PEO}$ adsorbs on the hydroxyl groups on the surface of the Si wafer, forming a copolymer brush. The conformation of the adsorbed molecules is different compared to that of molecules which are free in the overlying melt. It is believed that the origin of the autophobic behavior of PS-PEO copolymers is related to the difference in entropy between the different chain conformations of adsorbed and free copolymers. ${ }^{31}$ The film dewets, leaving behind a thin film of adsorbed copolymer and macroscopic drops.

After long annealing times, only droplets of the copolymer could be seen by optical microscopy on the substrate, as illustrated in Figure 7a. The copolymer microphase separates within the dewetted droplets, and both blocks are 
(c)

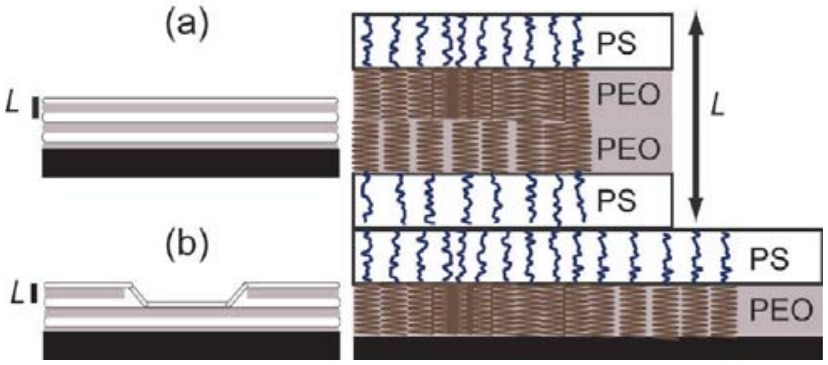

Figure 8. Schematic cross section of diblock copolymer thin film morphologies: lamellae parallel to the surface, typical of films of thickness (a) $h=\left(2 L+{ }^{1} / 2 L\right)$ and (b) $h$ just smaller than $\left(2 L+{ }^{1} / 2 L\right)$. The gray color represents the PEO block, and the white color the PS block. (c) Model for the arrangement of PS and PEO blocks at the top surface of a PS-PEO thin film.

present at the droplet's surface, as revealed by the phase contrast in the tapping mode AFM phase image in Figure $7 \mathrm{~b}$. Rounded domains of diameter $200 \pm 50 \mathrm{~nm}$ are visible inside the droplets. By comparison, the contrast between the rounded domains and the rest of the droplet is quite faint in the corresponding AFM height image (Figure S6), which means that the phase contrast is not due to topography but truly to differences in mechanical properties. Although assignment of bright and dark regions in phase images is not straightforward, ${ }^{46}$ the volume fraction of PS in the copolymer and the surface coverage of these rounded features suggests the formation of spherical or cylindrical domains of the minority block PS in a matrix of the majority block PEO.

The onset of dewetting in these PS-PEO thin films is delayed with respect to the dewetting observed in metastable homopolymer films of the same film thickness. ${ }^{68}$ It is likely that both the presence of the lamellar structure within the film and the crystallinity of the PEO block contribute to kinetically stabilize the film, so that the nucleation of the first holes occurs only on prolonged annealing. Studies are being conducted in our lab in order to characterize the intermediate stages between the initial microphase separation and the final dewetting.

\section{Discussion}

Tapping-mode AFM imaging revealed in an unmistakable way that only one block of the copolymer is present at the PS-PEO film's surface. The clear crystalline structure visible both in the AFM and optical micrographs suggested that the PEO block is very close to the surface and dominates the overall topography of the films. Condensation figures also showed no clear contrast in the wettability properties of different areas of the surface, confirming the presence of a single type of surface chemistry at the top surface of the film.

XPS analysis revealed that both the PS and the PEO blocks are present within the top $\sim 10 \mathrm{~nm}$ of the surface. The abrupt change of the XPS depth profile after $2 \mathrm{~s}$ of etching time suggests that the PS is at the top, that this PS-rich layer is a few nm thick, and that it is placed on top of a PEO layer (Figure 8). Contact angle measurements and AFM images of wetted films revealed that the PEO block is very close to the surface and can move rapidly to the very top of the film upon exposure to water.

The neutron reflectivity data provided the most conclusive evidence of the exact composition of the surface, indicating that the PS block is on the top surface of the film. In this study selective deuteration of one of the blocks was not necessary, because there is sufficient difference in the neutron scattering length densities of the PS and PEO blocks (respectively, $\sim 1.4 \times 10^{-6} \AA^{-2}$, and $\sim 0.7 \times 10^{-6} \AA^{-2}$, Table S1) to provide adequate scattering contrast between the individual phase-separated layers. This clearly enables us to assign either PS or PEO to distinct phaseseparated layers within the lamellar structure of the block copolymer film.

On the basis of the evidence from all the employed techniques, we conclude that the top surface of PS-PEO films spun from chloroform is made of only one PS block, which completes the PS-PEO lamellar stack. The presence of the PS layer does not mask the appearance of long-range crystalline structures due to $\mathrm{PEO}$, and also does not substantially alter the wettability properties of the PEO block. This arrangement effectively decreases the surface energy of the film by exposing to air the PS block, but maintaining the overall morphology of the crystalline PEO block. The crystallization kinetics of the PEO block controls the overall kinetics of microphase separation and the morphology of the final state, as in other systems. ${ }^{34-37}$

Our experiments compare well with the few existing studies performed on PS-PEO copolymers spun from chloroform, which found evidence of an enrichment of the PS block at the top surface in air. ${ }^{6,12,29,30}$ However, our experiments disagree with the interpretation of the data presented by Thomas and O'Malley, ${ }^{12}$ as we do not observe both blocks at the air/film interface. Reference 12 did consider the possibility for the presence of a thin PS overlayer, but the model was not consistent with their interpretation of the angular-dependent XPS data. There are a few possible explanations for this discrepancy. The first is the fact that in the previous study films were prepared on aluminum substrates, and were not annealed and therefore had not reached equilibrium. ${ }^{21}$ Therefore, the systems studied in ref 12 and here could be genuinely different. However, we need to point out that we did not observe any evidence of the presence of both blocks at the surface when imaging the as-spun films by tapping mode AFM. The second is that ref 12 used dip coating for film preparation, and so it is possible that the films were thicker than those studied here (no mention is made in the paper of the overall thickness). As the film thickness increases, it is possible that the parallel lamellar alignment is lost, and more random structures appear. It should be noted that the humidity was not controlled in either set of experiments, and it is not known whether this factor could affect microphase separation in thermally annealed films. Conversely, it is possible that the interpretation of the employed angular-dependent XPS data might change if the presence of island/hole features on the surface of the films or film thickness variations are taken into account.

Clear evidence of both PS and PEO blocks being present at the air/copolymer interface was observed by tapping mode AFM only in a second stage of the experiments, when the films were annealed for several hours and dewetted due to autophobic behavior into isolated droplets. At this final stage, rounded domains (either cylindrical or spherical) of the PS blocks could be observed within the PEO dewetted droplets.

\section{Conclusions}

A fundamental investigation on the nature of the surface of thin PS-PEO copolymer films spun from chloroform was performed, using several surface techniques, and the interpretation of results previously published was challenged. The use of multiple and complementary techniques allowed to shed some light on the elusive composition and topography of the top PS-PEO surface. Spontaneous dewetting processes in thin polymer films can be gainfully used to achieve micropatterned substrates to be used in biological applications. ${ }^{58}$ By exploiting the biocompatibility of PEO and the interplay between microphase separation and autophobic behavior in PS-PEO thin films, these systems 
could be employed to design surfaces with patterned biocompatibility for applications in biology and biotechnology.

Acknowledgment. C.N. thanks Dr. Tim Senden for providing the PS-PEO copolymer and acknowledges funding from the Australian Research Council.

Supporting Information Available: Figures showing and text discussing additional AFM and optical micrographs, differential scanning calorimetry, and thermal gravimetric analysis and a table of parameters used in the neutron reflectometry analysis. This material is available free of charge via the Internet at http:/ pubs.acs.org.

\section{References and Notes}

(1) Hamley, I. W., The Physics of Block Copolymers; Oxford University Press: New York, 2000.

(2) Bates, F. S.; Fredrickson, G. H. Annu. Rev. Phys. Chem. 1990, 41, $525-557$

(3) Keller, A.; Odell, J. A., In Processing, Structure and Properties of Block Copolymers; Folkes, M. J., Ed. Elsevier: London, 1985.

(4) Fasolka, M. J.; Mayes, A. M. Ann. Rev. Mater. Res. 2001, 31, 323355.

(5) Park, M.; Harrison, C.; Chaikin, P. M.; Register, R. A.; Adamson, D. H. Science 1997, 276, 1401-1404.

(6) Lin, Z. Q.; Kim, D. H.; Wu, X. D.; Boosahda, L.; Stone, D.; LaRose, L.; Russell, T. P. Adv. Mater. 2002, 14, 1373-1376.

(7) Kim, S. H.; Misner, M. J.; Xu, T.; Kimura, M.; Russell, T. P. Adv. Mater. 2004, 16, 226-231

(8) Tang, C.; Lennon, E. M.; Fredrickson, G. H.; Kramer, E. J ; Hawker, C. J. Science 2008, 322, 429-432.

(9) Harris, J. M.; Zalipsky, S. Poly (ethylene glycol): Chemistry and Biological Applications; American Chemical Society: Washington, DC, 1997; p 489.

(10) Lee, J. H.; Lee, H. B.; Andrade, J. D. Prog. Polym. Sci. 1995, 20, 1043-1079.

(11) Vanzo, E. J. Polym. Sci., A: Polym. Chem. 1966, 4, 1727-1730.

(12) Thomas, H. R.; O'Malley, J. J. Macromolecules 1979, 12, 323-329.

(13) O’Malley, J. J.; Thomas, H. R.; Lee, G. M. Macromolecules 1979, $12,996-1001$.

(14) Hasegawa, H.; Hashimoto, T. Macromolecules 1985, 18, 589-590

(15) Schmitt, R. L.; Gardella, J. A.; Magill, J. H.; Salvati, L.; Chin, R. L. Macromolecules 1985, 18, 2675-2679.

(16) Fredrickson, G. H. Macromolecules 1987, 20, 2535-2542.

(17) Henkee, C. S.; Thomas, E. L.; Fetter, L. J. J. Mater. Sci. 1988, 23, $1685-1694$.

(18) Coulon, G.; Russell, T. P.; Deline, V. R.; Green, P. F. Macromolecules 1989, 22, 2581-2589.

(19) Russell, T. P.; Coulon, G.; Deline, V. R.; Miller, D. C. Macromolecules 1989, 22, 4600-4606.

(20) Anastasiadis, S. H.; Russell, T. P.; Satija, S. K.; Majkrzak, C. F. Phys. Rev. Lett. 1989, 62, 1852-1855.

(21) Green, P. F.; Christensen, T. M.; Russell, T. P.; Jerome, R. J. Chem. Phys. 1990, 92, 1478-1482.

(22) Green, P. F.; Christensen, T. M.; Russell, T. P.; Jerome, R. Macromolecules 1989, 22, 2189-2194.

(23) Krausch, G. Mater. Sci. Eng. 1995, 14 (1-2), 1-94.

(24) Russell, T. P. Аnпи. Rev. Mater. Sci. 1991, 21, 249-268.

(25) Collin, B.; Chatenay, D.; Coulon, G.; Ausserre, D.; Gallot, Y Macromolecules 1992, 25, 1621-1622.

(26) Green, P. F.; Limary, R. Adv. Colloid Interface Sci. 2001, 94 (1-3), $53-81$.

(27) Grainger, D. W.; Okano, T.; Kim, S. W.; Castner, D. G.; Ratner, B. D.; Briggs, D.; Sung, Y. K. J. Biomed. Mater. Res. 1990, 24, 547571.

(28) Vulić, I.; Pijpers, A. P.; Okano, T.; Kim, S. W.; Feijen, J. J. Mater. Sci.: Mater. Med. 1993, 4, 353-365.

(29) Botelho do Rego, A. M.; Pellegrino, O.; Martinho, J. G.; Lopes da Silva, J. Surf. Sci. 2001, 482-485, (Part 2), 1228-1234.
(30) Botelho, A. M.; Pellegrino, O.; Martinho, J. M. G.; Lopes, J. Langmuir 2000, 16, 2385-2388.

(31) Reiter, G.; Vidal, L. Eur. Phys. J. E 2003, 12, 497-505.

(32) Gaines, G. L. Macromolecules 1981, 14, 208-208

(33) Clark, D. T.; Peeling, J.; O'Malley, J. M. J. Polym. Sci. Polym. Chem. Ed. 1976, 14, 543-551.

(34) Liang, G.-D.; Xu, J.-T.; Fan, Z.-Q.; Mai, S.-M.; Ryan, A. J. Macromolecules 2006, 39, 5471-5478.

(35) Reiter, G.; Castelein, G.; Hoerner, P.; Riess, G.; Sommer, J. U.; Floudas, G. Eur Phys. J. E 2000, 2, 319-334.

(36) De Rosa, C.; Park, C.; Lotz, B.; Wittmann, J.-C.; Fetters, L. J.; Thomas, E. L. Macromolecules 2000, 33 (13), 4871-4876.

(37) Hong, S.; MacKnight, W. J.; Russell, T. P.; Gido, S. P. Macromolecules 2001, 34, 2398-2399.

(38) Sherman, R.; Hirt, D.; Vane, R. J. Vac. Sci. Technol. A 1994, 12, $1876-1881$

(39) Bates, F. S.; Fredrickson, G. H. Phys. Today 1999, Feb, 32-38.

(40) Tsarkova, L.; Knoll, A.; Krausch, G.; Magerle, R. Macromolecules 2006, 39 (10), 3608-3615.

(41) Sriprom, W.; James, M.; Perrier, S.; Neto, C. Macromolecules 2009, 42,3138-3146

(42) Magonov, S. N.; Cleveland, J.; Elings, V.; Denley, D.; Whangbo, M. H. Surf. Sci. 1997, 389 (1-3), 201-211.

(43) Magonov, S. N.; Reneker, D. H. Anпu. Rev. Mater. Sci. 1997, 27 (1), 175-222.

(44) McLean, R. S.; Sauer, B. B. Macromolecules 1997, 30, 8314-8317.

(45) Knoll, A.; Horvat, A.; Lyakhova, K. S.; Krausch, G.; Sevink, G. J. A.; Zvelindovsky, A. V.; Magerle, R. Phys. Rev. Lett. 2002, 89, 035501.

(46) Wang, H.; Djurisic, A. B.; Chan, W. K.; Xie, M. H. Appl. Surf. Sci. 2005, 252, 1092-1100.

(47) Knoll, A.; Magerle, R.; Krausch, G. Macromolecules 2001, 34, $4159-4165$

(48) James, M.; Nelson, A.; Brule, A.; Schulz, J. C. J. Neutron Res. 2006, $14(2), 91-108$.

(49) Nelson, A. J. Appl. Crystallogr. 2006, 39, 273-276.

(50) Mansky, P.; Tsui, O. K. C.; Russell, T. P.; Gallot, Y. Macromolecules 1999, 32 (15), 4832-4837.

(51) Smith, A. P.; Douglas, J. F.; Meredith, J. C.; Amis, E. J.; Karim, A. Phys. Rev. Lett. 2001, 1, 8701.

(52) Massa, M. V.; Dalnoki-Veress, K.; Forrest, J. A. Eur. Phys. J. E 2003, 11 (2), 191-198

(53) Hong, S.; MacKnight, W. J.; Russell, T. P.; Gido, S. P. Macromolecules 2001, 34, 2876-2883.

(54) Sun, L.; Zhu, L.; Ge, Q.; Quirk, R. P.; Xue, C.; Cheng, S. Z. D. Hsiao, B. S.; Avila-Orta, C. A.; Sics, I.; Cantino, M. E. Polymer 2004, 45, 2931-2939.

(55) Izumi, K.; Ping, G.; Toda, A.; Miyaji, H.; Hashimoto, M.; Miyamoto, Y.; Nakagawa, Y. Jpn. J. Appl. Phys. 1994, 33, L1628-L1630.

(56) Iwanishi, M.; Harima, T.; Iijima, Y. Anal. Sci. 2001, 17 (ICAS2001), i391-i393.

(57) Jannasch, P. Macromolecules 1998, 31, 1341-1347.

(58) Neto, C. Phys. Chem. Chem. Phys. 2007, 9, 149-155.

(59) Kumar, A.; Whitesides, G. M. Science 1994, 263 (5143), 60-62.

(60) Reiter, G. Phys. Rev. Lett. 1992, 68 (1), 75-78.

(61) Seemann, R.; Herminghaus, S.; Neto, C.; Schlagowski, S. Podzimek, D.; Konrad, R.; Mantz, H.; Jacobs, K. J. Phys.: Condens. Matter 2005, 17, S267-S290.

(62) Neto, C.; Jacobs, K.; Seemann, R.; Blossey, R.; Becker, J.; Grün, G. J. Phys.: Condens. Matter 2003, 15, 3355-3366.

(63) Neto, C.; Jacobs, K.; Seemann, R.; Blossey, R.; Becker, J.; Grün, G. J. Phys.: Condens. Matter 2003, 15, S421-S426.

(64) Neto, C.; Jacobs, K. Physica A 2004, 339 (1-2), 66-71.

(65) Reiter, G.; Sommer, J.-U. J. Chem. Phys. 2000, 112, 4376-4383.

(66) Hamley, I. W.; Hiscutt, E. L.; Yang, Y.-W.; Booth, C. J. Colloid Interface Sci. 1999, 209, 255-260.

(67) de Gennes, P.-G.; Brochard-Wyart, F.; Quéré, D., Capillarity and Wetting phenomena. Drops, bubbles, pearls, waves. Springer: New York, 2004.

(68) Jacobs, K.; Herminghaus, S.; Mecke, K. R. Langmuir 1998, 14, 965-969. 